\title{
Human sensory nerve compound action potential amplitude: variation with sex and finger circumference
}

\author{
CHARLES F BOLTON AND KRISTINE M CARTER \\ From the Electromyography Laborutory, Department of Clinical Neurological Sciences, Victoria \\ Hospital, University of Western Ontario, London, Ontario, Canada.
}

SUMMARY The amplitude of human, antidromic, sensory compound action potentials (CAP) recorded from median and ulnar digital nerves is greater in females than males. This sex difference is probably due entirely to females having digits of smaller circumference, resulting in digital nerves being closer to the recording ring electrode enclosing the digit. The negative linear correlation between CAP amplitude and circumference holds true for persons of the same sex.

We have found that in studies of antidromic sensory conduction in median and ulnar nerves, the compound action potential (CAP) amplitude from digital nerves recorded with ring electrodes is greater in females than males. This may be due to digital nerves being closer to the skin surface in females, their digits being thinner, on the average, than males. In this study, we have assumed that finger or wrist circumference is proportionate to the distance between nerves and skin surface recording electrodes, and compared this circumference to the amplitude of sensory CAPs recorded in healthy males and females.

\section{Methods and materials}

Ten healthy males and 10 healthy females were studied; their ages (mean (range) years) were: 31 (23-43), 30 (23-60), respectively.

An inelastic tape measure, two centimetres wide with the end cut square at the zero line, was used to measure the finger and wrist circumferences at the level of the application of recording electrodes. Care was taken to keep tension on the tape the same for each measurement and to overlap completely the end of the tape except for a small margin allowing the circumference to be read. To test the accuracy of these measurements, they were repeated one month later and the differences were (mean

Address for reprint requests: Dr Charles F Bolton, Department of Clinical Neurological Services, Victoria Hospital, University of Western Ontario, London, Ontario, Canada.

Accepted 13 March 1980 (range $\mathrm{cm}$ ): wrist, $0.13(0.02-0.40)$; index finger $0.06(0.00-0.50)$; and little finger $0.04(0.02-0.30)$. The temperature (mean (range) ${ }^{\circ} \mathrm{C}$ ) was recorded distally in the palm with a skin surface thermistor: males-33.1 (31.0-34.8), and females-32.5 (31.0 34.0).

In antidromic sensory conduction studies, the nerves were stimulated by saline soaked pads spaced $2.5 \mathrm{~cm}$. apart. A Disa 14E11 constant voltage stimulating unit delivered pulses $0.1 \mathrm{~ms}$ in duration. Supramaximal stimuli excited the median and ulnar nerves at the wrist, and the superficial branch of the radial nerve in the lower forearm. The median and ulnar nerve CAPs were recorded with Teca ring electrodes at the index and little fingers, respectively, the recording electrodes being at the level of the proximal crease of the digit. The CAP of the superficial branch of the radial nerve at the wrist was recorded with Beckman miniature electrodes. In orthodromic conduction studies, supramaximal stimuli were delivered to the index and little fingers through Teca ring electrodes and recordings were made with Beckman miniature electrodes placed at the wrist over median and ulnar nerves, respectively.

Distances (mean (range) $\mathrm{cm}$ ) between recording and stimulating electrodes were: for females - median antidromic and orthodromic conduction $13.7(11.8-15 \cdot 1)$, ulnar antidromic and orthodromic conduction $11.2(9.9-12.5)$ and radial antidromic conduction 13.3 (12.5-13.8); for males $-15.6(14 \cdot 2-16.5), \quad 10.8(11 \cdot 8-13.5)$, and 14.5 (11.7-16.5), respectively. Recording and reference electrodes were spaced $3 \mathrm{~cm}$ apart and had an electrode impedance $<10 \mathrm{kohms}$ at $1000 \mathrm{~Hz}$. 
Evoked action potentials were transmitted to integrated circuit amplifiers, and were displayed on a Hewlett Packard 141B oscilloscope. The frequency range setting was $10 \mathrm{~Hz}$ to $2 \mathrm{kHz}$.

In orthodromic conduction studies, the amplitude of the sensory action potential was measured peak to peak. In antidromic conduction studies the amplitude was measured as the height of the negative wave since the late positive component of the sensory action potential in antidromic conduction may be distorted by muscle compound action potential. ${ }^{1}$

\section{Results}

We have reported a preliminary study with similar results. ${ }^{2}$ In the present study, the mean index finger, little finger and wrist circumferences of males were each larger than females (table). Mean values of median and ulnar nerve CAP

Table Circumferences ( $\mathrm{cm} \pm 1 \mathrm{SD})$

\begin{tabular}{lrrl}
\hline & Male $(10)$ & Fermale $(10)$ & $\mathrm{p}<$ \\
\hline Index finger & $7 \cdot 3 \pm 0.5$ & $6.4 \pm 0.4$ & 0.001 \\
Little finger & $6 \cdot 2 \pm 0.4$ & $5 \cdot 3 \pm 0.3$ & 0.001 \\
Wrist & $17.5 \pm 1.4$ & $15.4 \pm 0.7$ & 0.001 \\
\hline
\end{tabular}

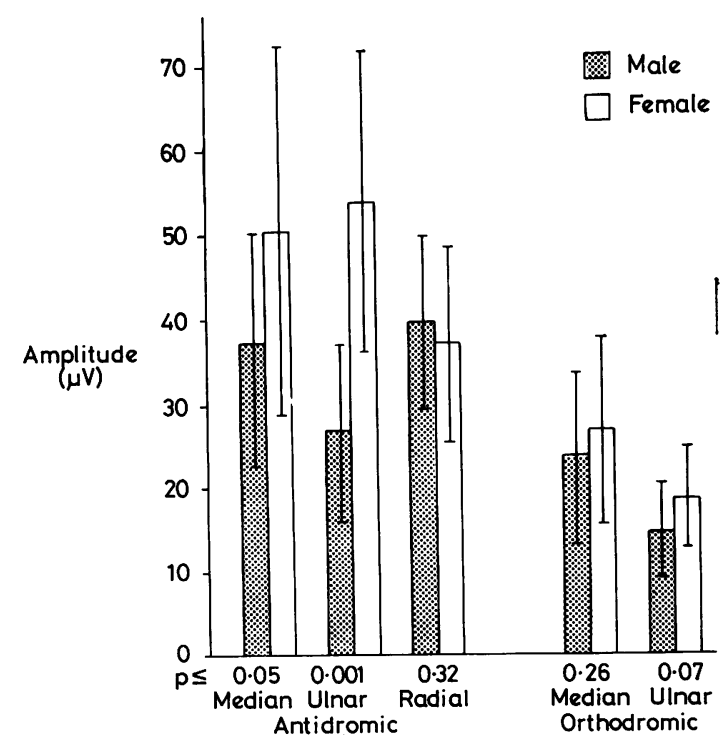

Fig 1 Sensory nerve CAP amplitudes were greater in females than males in median and ulnar antidromic studies. There was no statistically significant difference in the other studies, although mean CAP amplitudes were again greater in females than males in median and ulnar orthodromic conduction. (Vertical bars represent $\pm S D$ ).

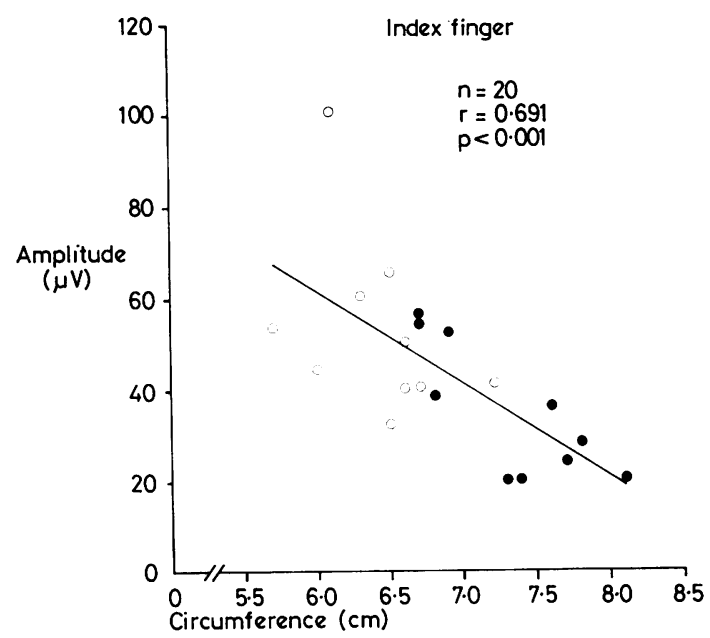

Fig 2 Median nerve antidromic sensory conduction study. The amplitude of the CAP recorded from digital nerves of the index finger on wrist stimulation showed a negative linear correlation with index finger circumference. Females (O) and males (O) formed relatively separate groups, suggesting this correlation is valid, irrespective of sex.

amplitudes on antidromic stimulation were much lower for males than for females (fig 1). Mean values of radial antidromic, and median and ulnar orthodromic, CAP amplitudes failed to show a statistically significant difference between the sexes. As noted previously by Buchthal and Rosenfalk, ${ }^{1}$ median and ulnar antidromic CAP amplitudes are greater than orthodromic.

The antidromic CAP amplitudes of median and ulnar nerves bore a statistically significant negative linear correlation with the circumference of index and little fingers, respectively (figs 2 and 3); males and females formed relatively separate groups. To determine if digit circumference was significantly related to CAP amplitude, irrespective of sex, further testing was done on healthy young subjects, 16 males and 16 females, of similar age. Such a relationship was shown for median sensory antidromic conduction in males (fig 4), and in females $(\mathrm{N}=16, \mathrm{r}=0.929, \mathrm{p}<0.001)$. No significant relationship was shown between wrist circumference and orthodromic median and ulnar, and antidromic radial, CAP amplitude.

\section{Discussion}

A number of variables may affect the amplitude of sensory CAPs recorded with surface electrodes: 


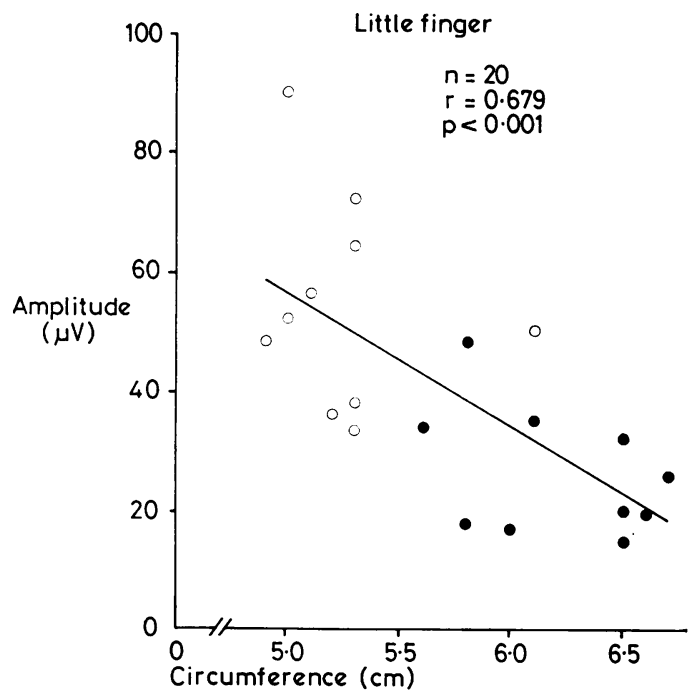

Fig 3 Ulnar nerve antidromic sensory conduction study. The results were similar to the median nerve study (fig 2).

the type of amplifier and its frequency range setting; the resistance of the recording electrode, of the space between the electrode and skin, and of the tissue between the nerve and recording electrode; the distance that the recording and reference electrodes are separated, CAP amplitude being attenuated if the distance is less than $2 \mathrm{~cm}^{13}$; and the temperature of the hand, lower temperature increasing CAP amplitude and duration. ${ }^{4}$ Amplitude decreases progressively with increasing distance between stimulating and recording electrodes due to dispersion resulting from varying conduction rates of sensory fibres. In our laboratory, there is an approximate $50 \%$ drop in amplitude from wrist to elbow for antidromic conduction. Buchthal and Rosenfalk ${ }^{1}$ noted a $75 \%$ drop in orthodromic conduction when using neer"e recording electrodes. The small differences in distance between males and females noted in our study (see Methods and materials) is unlikely to have significantly affected amplitude. Amplitude decreases in old age $^{1}$ but the males and females in our study were young, and of similar age.

Variations in the extent of cutaneous supply of the hand by median, ulnar and radial nerves ${ }^{5-7}$ will cause variations in the total number of nerve fibres in the digital, median, ulnar and superficial radial nerves, and, hence, in CAP amplitude recorded from these nerves. In pathological conditions, a decrease in either the density or total number of fibres will cause a reduction in CAP amplitude. ${ }^{8}$ Disproportionate slowing of conduction in remaining fibres will cause CAP dispersion and resulting amplitude reduction.

Our study showed in antidromic sensory, median and ulnar nerve conduction, that CAP amplitude was greater in females than males and that the circumference of digits in each sex, independently, showed a negative linear correlation with this amplitude. This sex difference may be explained by males having digits of greater circumference than females. Examination of the physical relationship between nerves and recording electrodes suggests the explanation. Four main nerve trunks transmitting the median nerve impulse are enclosed by the index finger ring electrode-two palmar digital nerves and the dorsal branch of each, and two main nerve trunks transmitting the ulnar nerve impulse are enclosed by the little finger ring electrode-two unbranched palmar digital nerves. ${ }^{5}$ Since electrical activity from these nerves is conducted in all directions and is likely to be recorded on all inner surfaces of the ring electrode, and since CAP amplitude varies negatively with distance between recording electrode and nerve, ${ }^{1}$ one would expect increasing circumference to be associated with the decreasing CAP ampitude shown in our study. This assumes the circumference is proportional to the various distances between the nerve and skin surface, an assumption that could only be proved by postmortem studies. The Beckman miniature

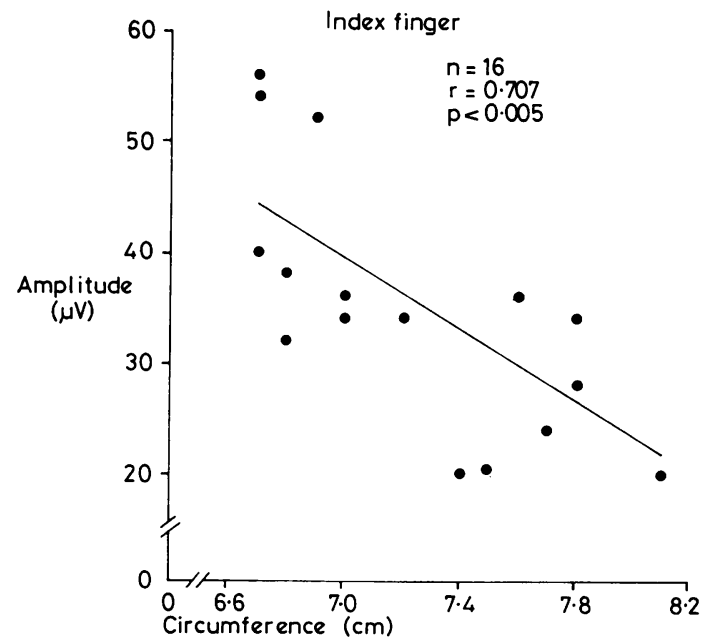

Fig 4 In males alone, CAP amplitude showed a negative linear correlation with digit circumference in median nerve antidromic sensory conduction studies; the results were similar for females. 
electrode placed on the wrist for recording median and ulnar orthodromic CAPs, and radial antidromic CAPs, is only $3 \mathrm{~mm}$ in diameter and encompasses only a small portion of the total wrist circumference. Thus, there was a poor relationship between CAP amplitudes recorded from these nerves and wrist circumference.

Our conclusions are based on the further assumption that the number of myelinated fibres in digital nerves is the same for both sexes. Studies performed to date on several sensory nerves-volar digital, ${ }^{9}$ anterior tibial ${ }^{1011}$ and sural ${ }^{12-16}$ - have shown considerable variation between individuals in the density and total numbers of myelinated fibres in a peripheral nerve, but no convincing differences between the sexes; further studies of digital nerves are needed to clarify this point. However, if the number of large myelinated nerve fibres in digital nerves is proportionate to the number of sensory receptors these fibres supply (which is unproven to our knowledge), results of a quantitative study of Meissner's corpuscles ${ }^{17}$ indirectly support our argument. This study showed that the density (number per $\mathrm{mm}^{2}$ of skin surface area) of these receptors in the glabrous skin of the little finger is greater in females than males, and varies negatively with the skin surface area of the little finger, suggesting the total number of Meissner's corpuscles is the same for each sex.

Our observations have practical importance in clinical electrophysiology. When interpreting antidromic sensory CAP amplitudes recorded with surface electrodes, the failure to take into account sex, and the circumference of the digit, may introduce considerable diagnostic error when comparing the results in an individual patient with control data that included both sexes. This would be particularly important in pathological conditions in which axonal degeneration predominates and maximum nerve conduction velocity remains near normal, the main effect being a reduction in CAP amplitude. ${ }^{8}$

We thank Mr Michael Clark for constructing EMG equipment, Miss Betsy Toth for typing the manuscript, and personnel in the Department of Clinical Neurological Sciences for volunteering as healthy subjects.

\section{References}

1 Buchthal F, Rosenfalk A. Evoked action poten- tials and conduction velocity in human sensory nerves. Brain Res 1966; 3:1-122.

2 Bolton CF, Carter KM. Amplitudes of sensory nerve action potentials in the hand recorded with surface electrodes. Electroenceph Clin Neurophysiol 1977; 43:599.

3 Buchthal F. Electromyography. In: Remond A et al, eds. Handbook of Electroencephalography and Clinical Neurophysiology. Amsterdam: Elsevier, 1973: 16:16B-20.

4 Bolton CF, Sawa GM, Carter K. Temperature effects on the size of human sensory compound action potentials. Can J Neuro Sci 1979; 6:378.

5 Hollinshead WH. The back and limbs. In: Anatomy for Surgeons. New York: HoeberHarper Book, 1958; 3:494-562.

6 Sayfi Y. Note sur l'innervation du dos de la main. Arch Anat Path 1968; A139-A140.

7 Sunderland $\mathrm{S}$. The median nerve anatomical features. In: Sunderland S, ed. Nerves and Nerve Injuries. Edinburgh: Churchill Livingstone, 1972: 754-8.

8 Lambert EH, Dyck PJ. Compound action potentials of sural nerve in vitro in peripheral neuropathy. In: Dyck PJ et al, eds. Peripheral Neuropathy. Philadelphia: WB Saunders, 1975; 1:430-1, 439.

9 Ranson SW et al. Number, size and myelination of the sensory fibres in the cerebrospinal nerves. In: Sensation: Its Mechanism and Disturbances. Ass Res Nerve Dis Proc 1935; 15:1-35.

10 Greenfield JG, Carmichael AE. The peripheral nerves in cases of subacute combined degeneration of the cord. Brain 1935; 58:483-91.

11 Swallow M. Fibre size and content of the anterior tibial nerve of the foot. J Neurol Neurosurg Psychiatry 1966; 29:205-13.

12 Sunderland $\mathrm{S}$ et al. The calibre of nerve fibres in human cutaneous nerves. J Comp Neurol 1949; 91:87-101.

13 Dyck PJ, Lambert EH. Numbers and diameters of nerve fibres and compound action potential of sural nerve: controls and hereditary neuromuscular disorders. Trans Amer Neuro Assoc 1966; 91:214-7.

14 O'Sullivan DJ, Swallow M. The fibre size and content of the radial and sural nerves. $J$ Neurol Neurosurg Psychiatry 1968; 31:464-70.

15 Ochoa J, Mair WGP. The normal sural nerve in man. I. Ultra-structure and number of fibres and cells. Acta Neuropath (Berl) 1969; 13:197216.

16 Gutrecht JA, Dyck PJ. Quantitative teased-fibre and histologic studies of human sural nerve during postnatal development. J Comp Neuro 1970; 138:117-29.

17 Bolton CF, Winkelmann RK, Dyck PJ. A quantitative study of Meissner's corpuscles in man. Neurology 1966; 16:1-9. 